\title{
Revealing Point Defects in a Large-Scale Scanning Diffraction Dataset
}

\author{
R. dos Reis ${ }^{1}$, C. Ophus ${ }^{1}$, J. Ciston ${ }^{1}$, P. Ercius ${ }^{1}$ and U. Dahmen ${ }^{1}$ \\ ${ }^{1 .}$ National Center for Electron Microscopy, Molecular Foundry, LBNL, Berkeley, CA, USA
}

The scattering of a finely focused electron beam traversing a thin foil provides rich information about the sample. By rastering the beam to record convergent beam electron diffraction (CBED) patterns at every position, the characteristics of a material can be probed to provide a detailed microstructural analysis. Scanning diffraction data offer the possibility of obtaining many types of images from a single experiment $[1,2]$. However, to extract relevant information from such large datasets requires efficient automated analytical tools. In this work we apply principal component analysis (PCA) to detect native oxygen point defects in zinc oxide ( $\mathrm{ZnO})$ [3] by distinguishing subtle intensity fluctuations at specific scattering angles within position-averaged CBED (PACBED) patterns. The two types of defect most likely to be present in this material are neutral and charged oxygen vacancies $\left(\mathrm{V}_{O}{ }^{\times}\right.$and $\left.\mathrm{V}_{O}{ }^{++}\right)$, both known to be important for luminescence. These defects are characterized by different displacements of the four $\mathrm{Zn}$ nearest neighbors - inward by $0.23 \AA$ for $\mathrm{V}_{\mathrm{O}}{ }^{\times}$or outward by $0.37 \AA$ for $\mathrm{V}_{\mathrm{O}}{ }^{++}[4]$.

Multislice simulations [5] of PACBED patterns were used to evaluate the scattering angles most sensitive to the presence of different type of point defects in the [1120] projection. Fig. 1(a) shows a simulated PACBED pattern divided into concentric rings for radial averaging at $1 \mathrm{mrad}$ intervals. The integrated intensity as a function of scattering angle is shown in Fig. 1(b), which compares the pattern for an ideal $\mathrm{ZnO}$ unit cell (black) to that for a unit cell containing a single defect, $\mathrm{V}_{\mathrm{O}^{\times}}$(red) and $\mathrm{V}_{\mathrm{O}}{ }^{++}$ (blue). Defects were placed in the middle of a $100 \AA$ thick slab. The presence of such a defect disturbs the patterns in characteristically different ways for charged and neutral vacancies. Because the differences are subtle, they are only visible in a plot of residual intensity $(\Delta \mathrm{I})$ obtained by subtracting the local pattern from the average (Fig1(c)). Defects placed at different depth $\mathrm{Z}$ result in different $\Delta \mathrm{I}$ values (shown shaded in Fig 1(c)) but maintain a characteristic pattern. Fig. 2(a) shows the PCA analysis applied to ideal $\mathrm{ZnO}$. Principal component \#1 is dominated by the edge of the central disc at $17 \mathrm{mrad}$, while higher components result from linear combinations of contributions at different scattering angles (mostly $<17 \mathrm{mrad}$ ). Fig. 2(b) presents the residual PCA variance, i.e. the difference between ideal $\mathrm{ZnO}$ and $\mathrm{ZnO}$ with oxygen vacancies. The residual of component \#1 is null since defects have a minimal effect on the edge of the disc. For higher components, \#2 and \#3, peaks at low scattering angles $<17 \mathrm{mrad}$ become apparent. The components are plotted on an adjusted scale for better visualization, as their weights decrease exponentially. Thus beyond component \#3, the data is irrelevant for this analysis.

Experimental datasets were recorded from $\mathrm{ZnO}$ nanowires by using a Gatan K2-IS direct electron detection camera installed on the TEAM I microscope [6] operated at $300 \mathrm{kV}$. Conventional high-angle annular dark field (HAADF) images (Fig 3a) were recorded concomitantly. The average dataset comprised 32,768 CBED patterns ( 0.25 Tb) covering an area of $20 \mathrm{~nm}$ x $10 \mathrm{~nm}$ ( 256 by 128 pixels). From this dataset, PACBED patterns were generated for each unit cell (inset in Fig. 3(a)). Our analysis then employed PCA to find the most significant variations in a matrix of the angular variable (0-35 mrad), vs observation (sequence of PACBED patterns). Components \#1, \#2 and \#3 are plotted in Fig. 3(b). While the edge dominates component \#1, components \#2 and \#3 show peaks at low scattering angles, as observed in the simulated data. Fig. 3(c) represents the variance of component \#2 for each unit cell within the analyzed region. This can be used as a criterion to select unit cells likely to contain a 
point defect, for example a high intensity point (red dot) surrounded by lower intensity neighbors. The PACBED can be further analyzed in order to distinguish the type of defect present. Fig 3(d) shows the residual radial average of specific pattern (A) compared to a neighbor (B). The residuals were obtained by subtracting the specific patterns from a mean PACBED of the whole (representing an ideal $\mathrm{ZnO}$ ). Despite experimental noise, the difference in the residual curve at lower angles remains detectable. Thus, our PCA based method allows us to distinguish point defects on the scale of unit cells in a large dataset obtained by scanning electron diffraction [7].

\section{References:}

[1] C. Gammer, V. B. Ozdol, C. H. Liebscher, and A. M. Minor, Ultramicroscopy 155, 1 (2015).

[2] V. B. Ozdol et al., Appl. Phys. Lett. 106, 253107 (2015).

[3] R. dos Reis, C. Ophus, J. Ciston, P. Ercius, and U. Dahmen, Microsc Microanal 21, 1097 (2015).

[4] A. Janotti and C. G. Van de Walle, Rep. Prog. Phys. 72, 126501 (2009).

[5] E. J. Kirkland, Advanced Computing in Electron Microscopy (Springer US, Boston, MA, 2010).

[6] R. Erni, M. D. Rossell, C. Kisielowski, and U. Dahmen, Phys. Rev. Lett. 102, 096101 (2009).

[7] Work at the NCEM/Molecular Foundry was supported by the Office of Science, Office of Basic Energy Sciences, of the U.S. Department of Energy under Contract No. DE-AC02-05CH11231. RR acknowledges the support of CAPES/BR Foundation Process No. 1204713-9.
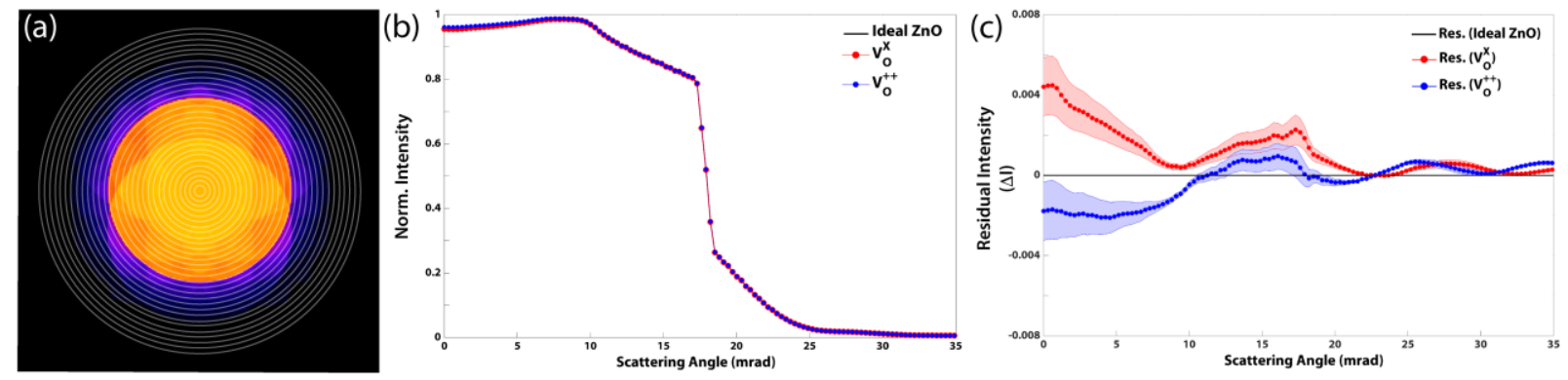

Figure 1. (a) Simulated PACBED pattern for a single unit cell showing concentric rings for radial averaging; (b) resulting plot of integrated intensity $v s$ scattering angle for ideal $\mathrm{ZnO}$ (black), a single $\mathrm{V}_{\mathrm{O}}{ }^{-}$-type (red) and $\mathrm{V}_{\mathrm{O}^{++}-}$ type (blue) point defect placed in the middle of a $100 \AA$ slab. (c) Residual intensity emphasizing the characteristic differences. Shaded curves represent the pattern variation for defects located at different $\mathrm{Z}$ heights.
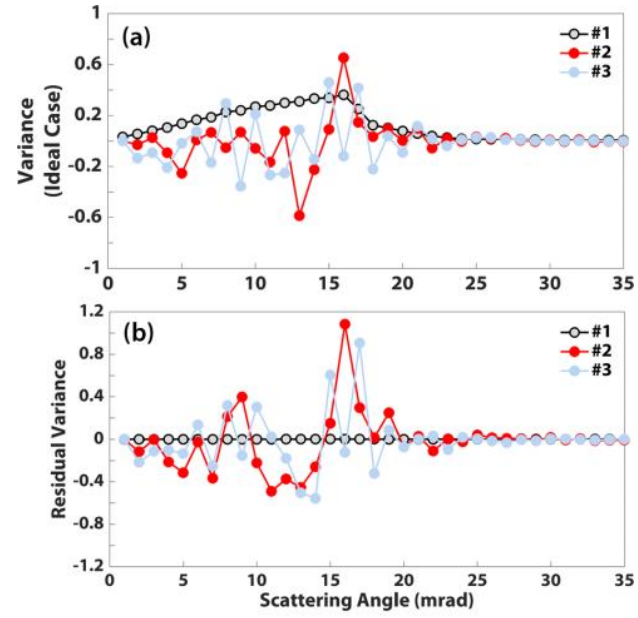

Figure 2. (a) Principal components \#1, \#2 and $\# 3$ in simulated PACBED patterns of ideal $\mathrm{ZnO}$. (b) PCA components of residual intensity comparing ideal $\mathrm{ZnO}$ to $\mathrm{ZnO}$ containing $\mathrm{V}_{\mathrm{O}} \times$ defects. Characteristic peaks in component \#2 (red) located at lower scattering angles can be related to defects.
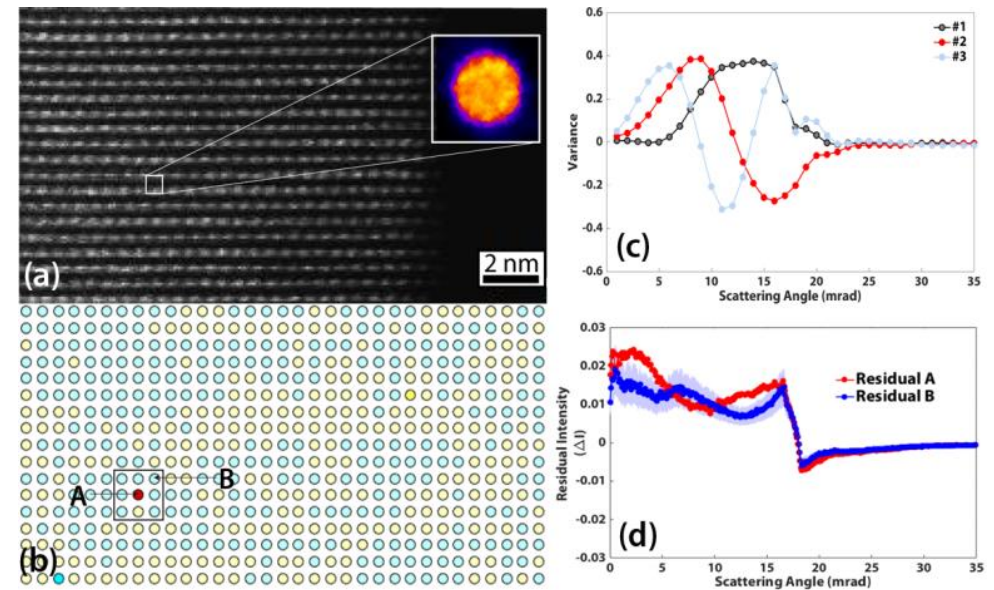

Figure 3. PCA analysis applied to experimental 4D-STEM dataset. (a) shows HAADF image. PACBED patterns (inset) can be extracted from each unit cell (white square). (b) plot of PCA components \#1, \#2 and \#3 extracted from the analysis of the whole dataset. (c) 2D-plot of intensity variance for component \#2. (d) Residual intensity comparing neighboring unit cells A - B. Shaded curve represents the average residual intensity around A. 